\title{
Leishmania (Viannia) braziliensis isolated from the saliva of patients in a cutaneous leishmaniasis-endemic area of northeastern Brazil
}

\author{
Maria Edileuza Felinto de Brito ${ }^{1 /+}$, Ericka Lima Almeida 1 , Angela Cristina Rapela Medeiros ${ }^{2}$, \\ Roberto Pereira Werkhäuser', Joanna Lucia de Almeida Alexandre', Bruna Santos Lima Figueiredo Sá1, \\ Eduardo Henrique Gomes Rodrigues', Sinval Pinto Brandão-Filho ${ }^{1}$
}

${ }^{1}$ Fundação Oswaldo Cruz-Fiocruz, Instituto Aggeu Magalhães, Departamento de Imunologia, Recife, PE, Brasil

${ }^{2}$ Universidade de Pernambuco, Recife, PE, Brasil

Several studies have described the use of non-invasive collection methods, mostly based on the detection of parasite DNA, for diagnosis. However, no Leishmania specimens have been isolated from saliva. Here, we report the first isolation of Leishmania braziliensis from the saliva of humans with cutaneous leishmaniasis but without lesions on their mucosa. The isolates were obtained from salivary fluid inoculated in hamsters and were tested by multilocus enzyme electrophoresis. Seven samples from 43 patients suspected of having the disease were identified for in vivo culture. These findings suggest that saliva is a clinical sample that allows the isolation of Leishmania sp.

Key words: Leishmania braziliensis - saliva - culture media

American cutaneous leishmaniasis (ACL) has a wide spectrum of clinical signs and symptoms, and many species of Leishmania are implicated as etiologic agents of this disease (Weigle \& Saraiva 1996). Clinical manifestations range from unapparent disease to skin lesions that may spontaneously resolve to multiple ulcerations and affected mucosa, which is associated with a high risk of disease recurrence and death (Costa et al. 2009).

Leishmania braziliensis is the primary and most prevalent pathogen associated with this disease in Brazil. This parasite is capable of producing the range of clinical manifestations, including cutaneous leishmaniasis (which may be spontaneously cured), mucosal leishmaniasis (with invasive ulcers), and disseminated cutaneous leishmaniasis (Costa et al. 2009). In a previous study, we detected 10 different kinds of zymodemes of L. (Viannia) braziliensis, which is the predominant species in vectors that circulate in Pernambuco, Brazil, in different clinical samples (Brito et al. 2009).

The isolation of promastigotes in culture medium allows the identification and characterisation of Leishmania species and therefore is of great epidemiological importance (Bensoussan et al. 2006, Rodríguez-González et al. 2006). Several studies have demonstrated non-invasive methods to collect biological samples for the diagnosis of leishmaniasis in patients suspected of having the disease (Mimore et al. 2002, Strauss-Ayali et al. 2004, Garcia et al. 2007, Figueroa et al. 2009, Boggild et al. 2011, Corvalan et al. 2011, Lombardo et al. 2012, Valencia et al. 2012). Al-

doi: 10.1590/0074-02760170250

+ Corresponding author: britomef@cpqam.fiocruz.br

Received 10 July 2017

Accepted 1 December 2017 though Corvalan et al. (2011) reported the presence of $L$. $(V$.) braziliensis DNA in the saliva of patients with skin lesions but without oral mucosa injury by polymerase chain reaction (PCR), they did manage to isolate the parasite from salivary fluid. Here, we report the first isolation of $L$. $(V$.) braziliensis from the salivary fluid of patients with ACL.

Multilocus enzyme electrophoresis (MLEE) is considered the gold standard for identifying Leishmania species. It allows the identification of isolates based on phenotypic polymorphisms of enzymes (Banuls et al. 2007, Schonian et al. 2010). The aims of this study were to (a) to isolate L. braziliensis from the saliva of patients with ACL and with no mucosal lesions, and (b) to evaluate the use of a non-invasive collection method for parasite isolation. The findings from this study suggest that saliva allows the isolation of Leishmania sp.

This study was performed in two municipalities: Moreno and Abreu e Lima, both of which belong to the metropolitan region of Recife, capital of Pernambuco state, Brazil. The two municipalities have no shared borders and are about $55.7 \mathrm{~km}$ from one another. The areas comprise $195.603 \mathrm{~km}^{2}$ and $125.99 \mathrm{~km}^{2}$ of land and have population densities of 311.94 inhabitants $/ \mathrm{km}^{2}$ and 785.69 inhabitants $/ \mathrm{km}^{2}$, respectively. The predominant economic activity in these regions is the cultivation of sugarcane. The houses are built close to remnants of the Atlantic Forest, with humans and domestic animals (horses, dogs, and cats) present. Lutzomyia whitmani is the vector primarily responsible for transmitting $L$. $(V$.) braziliensis in these municipalities (Brito et al. 2012).

In 2015, a total of 106 patients attending the dermatology outpatient clinic of Oswaldo Cruz Hospital, Recife, Brazil, were examined. Prior to sample collection, the participants provided informed consent using documents approved (protocol no. 038/11) by the Ethics Committee of Aggeu Magalhães Institute at the Oswaldo Cruz Foundation (AMI-PE). 
Salivary fluid samples were collected in a tube, with 5-mm samples of lesions obtained from the same patients. The biological samples were transported separately in a refrigerated box. One hundred microliters of saliva and macerated tissue fragments were inoculated into hamsters' peritonea at the Experimental Bioterium of IAM/FIOCRUZ-PE. Clinical samples (41 from tissue fragments and 43 with salivary fluid) from 106 patients were individually inoculated into 84 hamsters. After inoculation, hamsters were maintained in separate, properly identified cages with tags containing the inoculation date, name of patient, municipality of origin, and type of sample. All biological samples and syringes were also marked with the same identifiers. The animals were observed daily for ruffled or weight loss; ascites and/or death. These observations were recorded in detail.

The infected hamsters were observed for three months and then were euthanised under anaesthesia (60$100 \mathrm{mg} / \mathrm{kg}$ thiopental). Fragments of viscera (livers and spleens) of hamsters were removed and inoculated in Schneider's medium. After five days, the cultures were examined for the presence of parasites. Conventional tests (in vivo culture using hamsters, blood smears from hamster liver, blood smears, and PCR) were performed using other samples (biopsied tissues and swabs and scrapings from lesions). Identification of promastigotes was accomplished by MLEE, using specimens from the Leishmania collection of Oswaldo Cruz Foundation, Rio de Janeiro, Brazil (CLIOC) and the enzymes glucose-6-phosphate dehydrogenase (G6PDH, EC 1.1.1.49), 6-phosphogluconate dehydrogenase (6PGDH, EC 1.1.1.44), and isocitrate dehydrogenase (ICD, EC 1.1.1.42) (Cupolillo et al. 1994, Rodrigues et al. 2002). The use of animals in the study was approved by the Ethics Committee on the Use of Animals of IAM/FIOCRUZ-PE under protocol number 092/2015, according to law 2008 of 11,794 by the National Council for Animal Experimentation Control.

All patients (both male and female) who participated in the study were from areas with endemic ACL. Their ages varied, and they each presented only a single skin lesion. From seven saliva samples and 41 macerated lesion fragments obtained from 43 patients suspected of having ACL, 36 L. (V.) braziliensis isolates, including seven from saliva inoculated into culture medium, were obtained.

The seven patients from whom isolates were obtained from saliva were of both sexes, had an average age of 32.28 years, and each had a single skin lesion with an evolution period of approximately one to six months on the back of his/her hand, arm, or leg that measured 3.9 $\times 2.4 \mathrm{~cm}^{2}$ on average (Table). These isolates were characterised by MLEE and confirmed by PCR (Cupolillo et al. 1994, Rodrigues et al. 2002, Brito et al. 2009, Lee $\&$ Wong 2009). The results of PCR showed that the promastigotes isolated from salivary fluid, skin exudates, and tissue swabs were of the subgenus Viannia.

From the seven patients whose saliva was positive for L. (V.) braziliensis, biopsy material was also obtained. All patients responded well to treatment with N-methyl glucamine at a dose of 5 to $15 \mathrm{mg} / \mathrm{kg}$ /day after only one cycle of treatment for 20 days. They were monitored for 
seven months, and throughout this period they did not develop additional mucosal lesions.

Classical methods to diagnose ACL have limitations in their effectiveness and applicability. Therefore, for diagnosis, it is necessary to evaluate a patient's history, clinically suspect a case, and then obtain laboratory confirmation smear, culture and molecular techniques of the presence of Leishmania parasites (Rodrigues et al. 2002, Brito et al. 2012). As an alternative to invasive approaches, non-invasive methods for collecting clinical samples have been developed. They are intended to be simple, practical, and more acceptable to patients. Swabbing of exudate from ulcerated lesions is a non-invasive method to collect samples from patients suspected of having ACL, and this is considered a good method to obtain samples for PCR diagnosis (Mimori et al. 2002, Figueroa et al. 2009, Boggild et al. 2011, Lombardo et al. 2012, Valencia et al. 2012). This approach allows the identification of Leishmania species in sub-clinical cases of disease in individuals with low parasitic burdens during treatment follow-up and to distinguish between current and past infections (Lombardo et al. 2012).

Lee \& Wong (2009) showed the advantages of using saliva as a diagnostic sample for surveillance of ACL. Early detection of Leishmania DNA in saliva can be beneficial, both for the initiation of treatment and for research purposes. Corvalan et al. (2011) detected $L$. (V.) braziliensis DNA by PCR of saliva from patients with mucosal lesions. They concluded that this was a new, simpler option for parasitological surveys. Figueroa et al. (2009) collected swab samples of the nasal mucosa, tonsils, and conjunctiva of patients with cutaneous and mucocutaneous leishmaniasis and identified Leishmania panamensis, L. braziliensis, and Leishmania guyannensis. They also showed that the amount of Leishmania DNA in saliva decreased with treatment (Phumee et al. 2013). Saliva can also be used to detect infections with Leishmania species (Siriyasatien et al. 2016). However, none of these studies isolated Leishmania parasites from salivary fluid.

Our finding that L. braziliensis can be isolated from saliva might help in elucidating the escape mechanisms and/or tropism of the parasite. Further studies are needed to evaluate the performance of various methods to obtain clinical samples, including saliva, from individuals with ACL. Saliva collection in this study was effective, fast, and well accepted by patients. Furthermore, saliva has great potential for use in paediatric populations.

\section{ACKNOWLEDGEMENTS}

To Dr Juliana Figueirêdo da Costa Lima Suassuna Monteiro for her valuable contribution to the final version of this manuscript.

\section{AUTHORS' CONTRIBUTION}

MEFB and EHGR conceived and designed the study; ACRM is the dermatologist who evaluated the patients; MEFB, ELA, and JLAA analysed and interpreted the data; MEFB and BSLFS wrote the paper; SPBF and RPW revised the manuscript; and SPBF and EHGR are guarantors of the study. All authors participated in conducting the study, had full access to the data, and read and approved the final manuscript.

\section{REFERENCES}

Banuls AL, Hide M, Prugnolle F. Leishmania and the leishmaniasis: a parasite genetic update and advances in taxonomy, epidemiology and pathogenicity in humans. Adv Parasitol. 2007; 64: 1-109.

Bensoussan E, Abedelmajeed N, Jonas F, Lionel FS, Jaffe CL. Comparison of PCR assays for diagnosis of cutaneous leishmaniasis. J Clin Microbiol. 2006; 44(4): 1435-9.

Boggild AK, Valencia BM, Veland N, Ramos AP, Calderon F, Low DE, et al. Nono-invasive cytology brush PCR diagnostic testing in mucosal leishmaniasis: superior performance to convencional biopsy whit histopatology. PLoS ONE. 2011; 6(10): 1-6.

Brito ME, Andrade MS, Dantas-Torres F, Rodrigues EH, Cavalcanti MP, Almeida AM, et al. Cutaneous leishmaniasis in northeastern Brazil: a critical appraisal of studies conducted in state of Pernambuco. Rev Soc Bras Med Trop. 2012; 45(4): 425-9.

Brito MEF, Andrade MS, Mendonça MG, Silva CJ, Almeida EL, Lima BS, et al. Species diversity of Leishmania (Viannia) parasites circulating in an endemic area for cutaneous leishmaniasis located in the Atlantic rainforest region of northeastern Brazil. Trop Med Int Health. 2009; 14(10): 1278-86.

Corvalan FH, Sampaio RNR, Brustoloni YM, Andreotti R, Lima Júnior MSC. DNA identification of Leishmania (Viannia) braziliensis in human saliva from a patient with American cutaneous leishmaniasis. J Venom Anim Toxins incl Trop Dis. 2011; 17(1): 98-102.

Costa JML, Saldanha ACR, Nascimento D, Sampaio G, Carneiro F, Lisboa E, et al. Clinical modalities, diagnosis and therapeutic approach of the tegumentary leishmaniasis in Brazil. GM Bahia. 2009; 79(3): 70-83.

Cupolillo E, Grimaldi Jr G, Momen H. A general classification of NewWorld Leishmania using numerical zymotaxonomy. Am J Trop Med Hyg. 1994; 50(3): 296-311.

Figueroa RA, Lozano LE, Romero IC, Cardona MT, Prager M, Pacheco R, et al. Detection of Leishmania in unaffected mucosal tissues of patients with cutaneous leishmaniasis caused by Leishmania (Vannia) species. J Infect Dis. 2009; 200(46): 638-46.

Garcia AL, Parrado R, de Doncker S, Bermudez H, Dujardin JC. American tegumentary leishmaniasis: direct species identification of Leishmania in non-invasive clinical samples. Trans R Soc Trop Med Hyg. 2007; 101(4): 368-71.

Lee YH, Wong DT. Saliva: an emerging biofluid for early detection of diseases. Am J Dent. 2009; 22(4): 241-8.

Lombardo, G, Pennisi MG, Lupo T, Migliazzo A, Capri A, SolanoGallego L. Detection of Leishmania infantum DNA by real-time PCR in canine oral and conjunctival swabs and comparison with other diagnostic techniques. Vet Parasitol. 2012; 184(1): 10-7.

Mimori T, Matsumoto T, Calvopiña MH, Gomesz EA, Sava H, Katakura K, et al. Usefulness of sampling with cotton swab for PCR-diagnosis of cutaneous leishmaniasis in the New World. Acta Trop. 2002; 81(3): 197-202.

Phumee A, Kraivichian K, Chusri S, Noppakun N, Vibhagool A, Sanprasert $\mathrm{V}$, et al. Detection of Leishmania siamensis DNA in saliva by polymerase chain reaction. Am J Trop Med Hyg. 2013; 89(5): 899-905

Rodrigues EH, Lombardo G, Pennisi MG, Lupo T, Migliazzo A, Capri A, et al. Detection of Leishmania infantum DNA by real-time PCR in canine oral and conjunctival swabs and comparison with other diagnostic techniques. Vet Parasitol. 2002; 184(1): 10-7.

Rodríguez-González I, Marín C, Vargas F, Córdova O, Barrera M, Gutiérrez-Sánchez R, et al. Identification and biochemical characterization of Leishmania strains isolated in Peru, Mexico, and Spain. Exp Parasitol. 2006; 112(1): 44-51. 
Schonian G, Mauricio I, Cupolillo E. Is it time to revise the nomenclature of Leishmania? Trends Parasitol. 2010; 26(10): 466-9.

Siriyasatien P, Chusri S, Kraivichian K, Jarivapan N, Hortiwakul T, Silpapoiakul K, et al. Early detection of novel Leishmania species DNA in the saliva of two HIV-infected patients. BMC Infect Dis. 2016; 16: 89 .

Strauss-Ayali D, Jaffe CL, Burshtain O, Goen L, Baneth G. Polymerase chain reaction using noninvasively obtained samples, for the detection of Leishmania infantum DNA in dogs. J Infect Dis. 2004; 189(9): 1729-33.

Valencia AM, Veland N, Alba M, Adaui V, Arevalo J, Low DE, et al. Nono-invasive cytology brush PCR diagnostic and causative species identification of American cutaneous leishmaniasis in Peru. PLoS ONE. 2012; 7(11): 1-6.

Weigle K, Saraiva NG. Natural history, clinical evolution, and the host-parasite interaction in New World cutaneous leishmaniasis. Clinic Dermatol. 1996; 14(5): 433-50. 